\title{
Percutaneous tracheostomy in COVID-19 pneumonitis patients requiring prolonged mechanical ventilation: Initial experience in 51 patients and preliminary outcomes.
}

\author{
Arunjit Takhar ${ }^{1}$, Chrysostomos Tornari ${ }^{1}$, Nikul Amin ${ }^{1}$, Duncan Wyncoll ${ }^{1}$, Stephen \\ Tricklebank $^{1}$, Asit Arora ${ }^{1}$, Imran Ahmad ${ }^{1}$, RICARD SIMO $^{1}$, and Pavol Surda ${ }^{1}$ \\ ${ }^{1}$ Guy's and Saint Thomas' NHS Foundation Trust
}

May 6, 2020

\section{Key Points}

- In 51 patients with COVID-19, the median day of tracheostomy after intubation was $17.72 .5 \%$ occurred on or after day 14. The average PEEP was 8 and Fi02 0.35.

- Median follow-up was 11 days (range (2-36)), out of which the sedation and ventilation was stopped in $98 \%$ and $88.2 \%$, respectively. Moreover, $23.5 \%$ patients were discharged from ICU and $17.6 \%$ decannulated. The mortality rate was $3.9 \%$; deaths and none were tracheostomy related.

- All ENT and most Intensivist operators used enhanced PPE with PAPR.

- None of the surveyed healthcare workers were clinically or PCR-test positive for COVID-19 in the relevant 5-14-day window post-procedure.

- We present an airway management algorithm for percutaneous tracheostomy utilising ultrasound and bronchoscopy to reduce the risk of intra-operative cuff puncture or misplacement, whilst minimising aerosol generation.

\section{Key Words}

SARS-CoV-2, Novel Coronavirus, COVID-19, Tracheostomy, Ventilator Weaning, Personal Protective Equipment (PPE), Airway Management

\section{Introduction}

The role of tracheostomy for weaning ventilated patients with COVID-19 pneumonitis remains a matter of debate. Controversy exists regarding timing, location, prognosis, techniques to reduce aerosol generation, and risk of transmission to healthcare workers. The mortality rate following admission to critical care with COVID-19 pneumonitis is currently higher than for non-COVID viral pneumonia (50.7\% vs $22.0 \%$ ) as described in the Intensive Care National Audit and Research Centre (ICNARC) report [1]. This must be factored into the decision making process.

ENT-UK currently recommended performing tracheostomy on or after 14 days of intubation [2], and the British Laryngological Association (BLA) recommend deferring until the patient has a positive end-expiratory pressure (PEEP) requirement of [?] $10 \mathrm{~cm} \mathrm{H}_{2} 0$ and an Fi02 of [?] 0.4 [3]. As yet, there is no evidence-base beyond expert consensus. 
Current evidence does not suggest a difference between the outcomes of surgical tracheostomy (ST) and percutaneous tracheostomy (PT) with regard to patient safety and aerosol-generation [4]. Recently some international guidelines have recommended a percutaneous approach [5-6].

Guy's \& St Thomas' NHS Foundation Trust (GSTT) was one of the first centres in the UK to treat COVID19 patients, and hence make decisions about tracheostomy. As of $24^{\text {th }}$ April 2020 there were 171 completed critical care admissions, with a mortality rate of 33\%. A further 104 are currently receiving intensive care for COVID-19 pneumonitis. In March 2020 we developed a dedicated ENT-led tracheostomy team, through close collaboration with intensive care and anaesthetics. Indications for tracheostomy were agreed, intensive training and simulation was undertaken, and our standardised protocol and recommendation is described in detail in our recent publication [7].

The aim of this paper is to analyse the intra-operative and short-term outcomes associated with tracheostomy for COVID-19 pneumonitis.

\section{Materials and Methods}

\subsection{Study population 85 setting}

In this prospective study we included adults ([?]18 years) undergoing elective tracheostomy between $21^{\text {st }}$ March $-24^{\text {th }}$ April 2020 for weaning of mechanical ventilation who had a confirmed diagnosis of COVID19 pneumonitis at GSTT. All procedures were performed by the intensive care team or dedicated COVID-19 ENT tracheostomy team. The study was registered and approved as clinical audit.

\subsection{Study objectives and measurements}

We analysed procedure timing and levels of ventilatory support at time of tracheostomy (PEEP [?] 10 or Fi02 [?] 0.5). We also documented surgical approach and duration, procedural complications, outcomes, mortality, and staff safety.

Patient demographics included age, gender, ethnicity, BMI, very severe co-morbidities, and APACHE II score. We compared our cohort to the ICNARC one that underwent advanced respiratory support during their critical care admission [1]. We measured ventilator settings (PEEP, Fi02) between 7am and 9am on the day of the procedure.

Outcome measures were recorded from day of tracheostomy until the day when:

* Sedation stopped: Intravenous sedative infusions (Propofol, Fentanyl, Alfentanil or Midazolam) successfully held for 24 hours

* Ventilation stopped: No mechanical ventilation required (including BiPAP / CPAP) for at least 24 hours

* Patient discharged from ITU

* Decannulation of tracheostomy

* Survival 
We separately analysed the outcomes of those who underwent tracheostomy on day $<14$ vs [?]14.

For the study duration, all team members involved were surveyed and asked to report COVID-19 symptoms [8], and volunteer whether they had COVID-19+ PCR test. Results were included if this occurred within 5-14 days after a procedure.

Descriptive data is presented and analysed. Tests for significance were performed using a Chi-Squared test and Mann-Whitney U Tests.

\section{Results}

\subsection{Patient demographics}

53 patients (19.3\% of critical care admissions) underwent tracheostomy but 2 did not meet inclusion criteria. One patient had negative COVID-19 screening and the second was tracheostomised due to cerebral infarcts. 51 patients were included for further analysis. Demographics were compared to ICNARC and presented in Figure 1 \& Table 1.

\subsection{Timing of tracheostomy}

The median (IQR) time to tracheostomy was 17 (13-20) days. 37 (72.5\%) of tracheostomies were performed on or after day 14 .

\subsection{Ventilatory $\& 3$ other organ support on day of procedure}

$49(96.1 \%)$ patients required PEEP of [?]10 $\mathrm{cmH}_{2} 0$ and 48 (94.1\%) were on a Fi02 of [?]0.5. The highest PEEP and Fi02 requirements were $12 \mathrm{cmH}_{2} 0$ and 0.55 respectively. 15 (29.4\%) were receiving vasopressors or inotropes, 15 (29.4\%) renal replacement therapy and 7 (13.7\%) were on ECMO.

\subsection{Teams, technique location \&3 complications}

48 (94.1\%) were performed as PT, and the remainder via hybrid technique. 50 (98.0\%) were performed at the bedside on an open ICU or side room. The mean (SD) procedure time $(\mathrm{n}=10)$ was $59.2+/-9.54$ minutes, which includes time for donning, doffing, kit preparation, safety checks and briefing. One patient required elective transfer to the operating theatre where a hybrid approach was performed, this was due to morbid obesity and anticipated difficult upper airway. 5 (9.8\%) intraoperative complications were recorded (table 1). There were no related oxygen desaturations. Four of these patients have successfully weaned off sedation and ventilation, and three have been decannulated; without sequelae to date. Only pharmacological interventions were required for post-operative bleeding.

\subsection{Outcomes and Mortality}

14-day follow-up data was available for 24 patients; we also present average times to each outcome measure for the whole study group with a median (IQR) follow up time of $11(6-16)$ days (Tab 1 \& Fig 2). 
We performed subgroup analysis of patients who underwent tracheostomy on day <14 (14 patients) vs [?]14 (37 patients) since intubation. Statistical analysis did not show significant difference between follow-up times. Moreover, outcomes including stopping sedation and ventilation, discharge from ITU and decannulation did not differ.

The mortality rate is presently $3.9 \%$ (2/51). Deaths occurred on day 9 and 14 post tracheostomy. The cause of death was ventilator dependant respiratory failure for both; one patient was on ECMO at the time of death.

\subsection{Staff safety}

All ENT surgeons wore powered air purifying respirators (PAPRs). Overall $87 \%$ of operators and $28 \%$ of other team members wore PAPRs, the remainder wore FFP3 mask and shield visor. Everyone wore double layer fluid-repellent disposable surgical gown and gloves.

There were a total of 67 healthcare workers involved in these procedures. Of those surveyed, none developed COVID-19 symptoms. The response rate was $83.8 \%$. Four reported having COVID-19 prior to undertaking the procedures.

\section{Discussion}

Despite a huge effort to produce guidelines to support decision-making and procedure for tracheostomy in COVID-19 patients, at present there has been no report on associated outcomes.

Our case mix appears to be a representative sample of patients in ICU's across the country; differences in ethnicity and BMI may be accounted for by our local population. This study includes some of the most critically ill COVID-19 patients, with a significant proportion having support of two or more organ systems, and a very high proportion receiving ECMO.

There was good adherence to departmental [7] and ENT-UK/BLA [2-3] guidelines with regards to timing and levels of ventilatory support in this study. As such, this preliminary analysis may give an indication of the expected outcomes this guidance might deliver.

We compared those who underwent tracheostomy before and after day 14 and observed no difference in outcomes. It appears that timing may not be the single factor that determines decision to proceed with tracheostomy and the broader clinical picture, including levels of ventilatory support must be carefully considered.

The obvious value of our bedside team-based approach has been to optimise resource utilisation during hugely challenging times. The mean procedure time was 59.2 minutes including donning, doffing, kit preparation, safety checks and briefing. It has been demonstrated that the need to transfer patients to theatre can be safely avoided in most cases. 
Complication rates for ENT surgeon-performed PT were comparable to ICU operators in our institution and existing published literature [9], including open techniques [10]. Misplacement (1 ENT, 1 ICU) rates were higher than previously published (1.5\%) [9], which may be due to small study size, but could reflect early attempts to avoid bronchoscope usage in order to minimise aerosol generation [6], as such, our protocols were adopted to mitigate these risks [7].

Cuff puncture also presented a particular concern for us due to risk of aerosol generation and potential for prolonged loss of ventilation. This highlighted an important point that cuffs for endotracheal tubes used on ICU are longer than commonly used in the theatre setting. In response, our anaesthetic colleagues, who are now increasingly providing airway management during procedures, have developed a specific action card (Figure 4), utilising real-time ultrasound to minimise risk. A standardised protocol, modified processes in response to early learning, and a multi-disciplinary approach have enabled us to safely deliver this service.

Our preliminary outcomes give a useful indication of the expected recovery course for COVID-19 tracheostomy patients, which is currently an unreported population. The preliminary mortality figure will rise with a longer follow-up period, but is still likely to be lower than the $31.5 \%$ 30-day mortality reported in a large trial of patients undergoing prolonged mechanical ventilation [9]. At present this study suggests that outcomes may be favourable when current guidance is adhered to.

Whilst there is still not consensus on which technique is less aerosol generating [4,5,6], our standards of personal protective equipment (including PAPRs) currently appear safe, with no identified healthcare worker transmission, reflective of other reports of PAPRs for tracheostomy [4].

Our early data can aid planning as we enter a phase of safe decannulation and rehabilitation. Longer-term follow up data on outcomes and late complications of tracheostomy are also needed before we can draw any further conclusions from this study.

The evidence we present to date would support the current national guidance as being appropriate, but we would emphasise the importance of clinical indication along with timing of procedure. The limitations of this study are the sample size and follow-up time; however it does represent a high-volume, prospective study completed in a short time frame, against a defined protocol. A co-ordinated national effort, as is being delivered by the ENT-UK COVID-19 tracheostomy audit will provide far more comprehensive evidence, and no doubt define the standards of care in the near future.

\section{References}

1. Intensive Care National Audit \& Research Centre.ICNARC report on COVID-19 in critical care. 2020. https://www.icnarc.org/Our-Audit/Audits/Cmp/Reports. Accessed 25 April 2020

2. ENT-UK. COVID-19 tracheostomy guidance. 2020.https://www.entuk.org/covid-19-tracheostomyguidance-t-jacob-et-al. Accessed 23 April 2020

3. British Laryngological Society. Tracheostomy Guideline. 2020. https://www.britishlaryngological.org/news/tracheostomy-guideline-covid-19. Accessed 23 April 2020 
4.Tay JK, Khoo ML, Loh WS. Surgical Considerations for Tracheostomy During the COVID-19 Pandemic: Lessons Learned From the Severe Acute Respiratory Syndrome Outbreak. JAMA Otolaryngol Head Neck Surg. 2020. [Published online 31 March 2020]. doi:10.1001/jamaoto.2020.0764

5. Schultz P, Morvan J, Fakhry N, et al. French consensus regarding precautions during tracheostomy and post-tracheostomy care in the context of COVID-19 pandemic. European Annals of Otorhinolaryngology, Head and Neck Diseases. 2020. [Published online 9 April 2020] doi: https://doi.org/10.1016/j.anorl.2020.04.006.

6. Brett A. Miles, DDS, MD. Tracheostomy During the COV-SARS-CoV-2 pandemic: Recommendations from the New York Head and Neck Society. Authorea. April 17 2020. DOI: 10.22541/au.158715705.50810853

7. Takhar, A., Walker, A., Tricklebank, S. et al. Recommendation of a practical guideline for safe tracheostomy during the COVID-19 pandemic. Eur Arch Otorhinolaryngol. 2020. [published online 21 April 2020] https://doi.org/10.1007/s00405-020-05993-x

8. Centers for Disease Control and Prevention. Coronavirus Disease 2019 COVID-19. 2020.https://www.cdc.gov/coronavirus/2019-ncov/symptoms-testing/symptoms.html Accessed 25 April 2020

9. Young D, Harrison DA, Cuthbertson BH, Rowan K, TracMan Collaborators FT. Effect of Early vs Late Tracheostomy Placement on Survival in Patients Receiving Mechanical Ventilation: The TracMan Randomized Trial. JAMA. 2013;309(20):2121-2129. doi:10.1001/jama.2013.5154

10. Lipton G, Stewart M, McDermid R, et al. Multispecialty tracheostomy experience. Annals of the Royal College of Surgeons of England. 2020 1:1-5. doi: 10.1308/rcsann.2019.0184

\section{Figure Legends}

Figure 1.Patient demographics and cumulative trends in tracheostomy

Table 1. Patient demographics, timing, complications and outcomes

Figure 2. Outcomes for first 14 days after tracheostomy

Figure 3 - Anaesthetic action card

\section{Supporting information (online only)}

Percutaneous tracheostomy action card
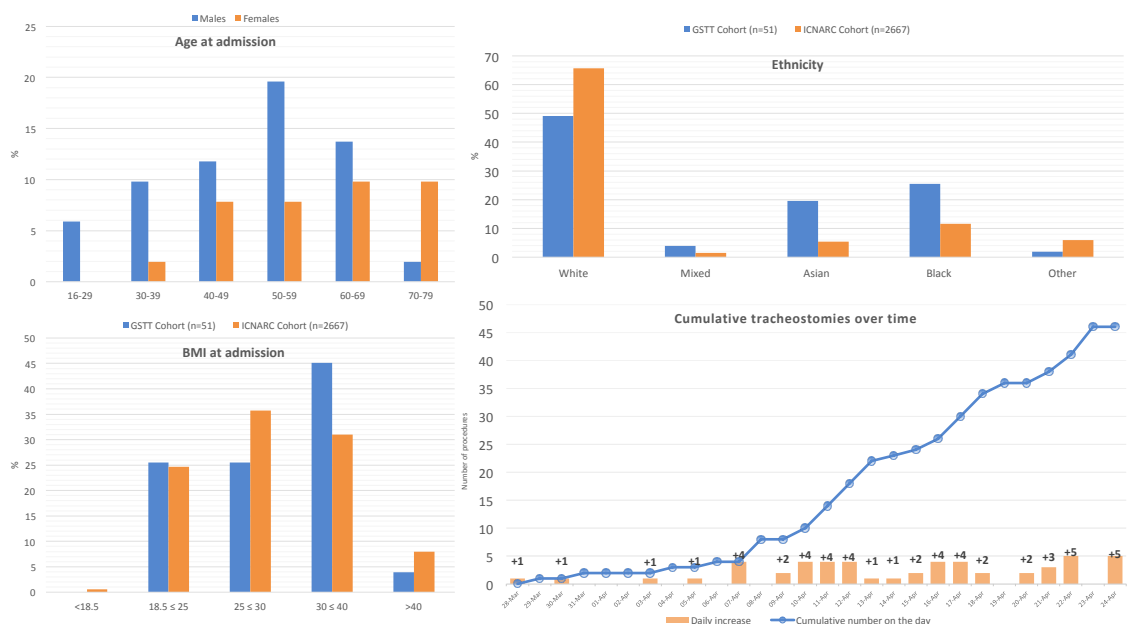


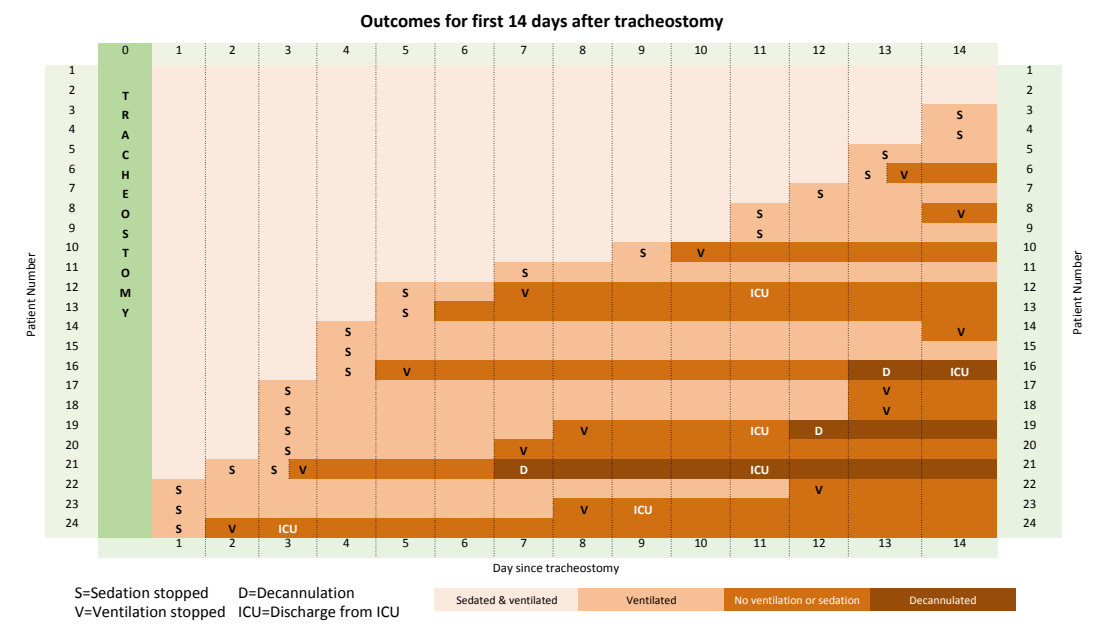

\section{Hosted file}

Table 1.pptx available at https://authorea.com/users/315585/articles/445924-percutaneoustracheostomy-in-covid-19-pneumonitis-patients-requiring-prolonged-mechanicalventilation-initial-experience-in-51-patients-and-preliminary-outcomes

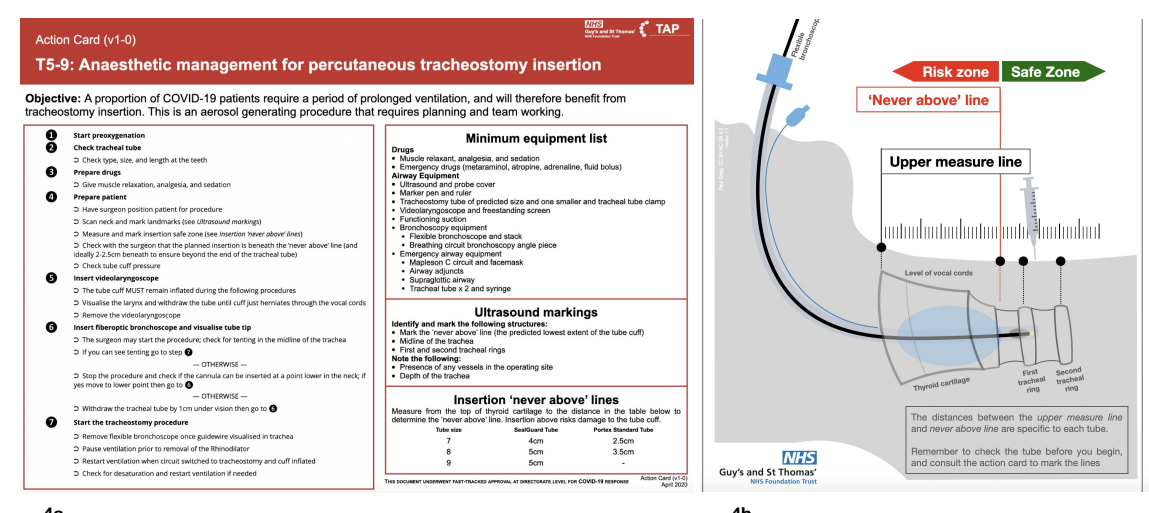

$4 a$

$4 b$ 